\title{
How do Somatic Markers Feature in Decision Making?
}

\author{
Jordan Bartol \\ University of Leeds \\ Stefan Linquist \\ University of Guelph
}

\begin{abstract}
Several recent criticisms of the somatic marker hypothesis (SMH) identify multiple ambiguities in the way it has been formulated by its chief proponents. Here we provide evidence that this hypothesis has also been interpreted in various different ways by the scientific community. Our diagnosis of this problem is that SMH lacks an adequate computational-level account of practical decision making. Such an account is necessary for drawing meaningful links between neurological- and psychological-level data. The paper concludes by providing a simple, five-step model of practical decision making. Recasting SMH in terms of this model generates more precise and empirically tractable computational-level hypotheses about the various ways that somatic markers might influence practical decisions.
\end{abstract}

\section{Introduction}

The somatic marker hypothesis (SMH) is a popular neuropsychological theory about the role of emotions in practical decision making. It has been highlighted as among the few successful examples of a "cross-level link" between psychological and neurological levels of description (Sun, Coward, \& Zenzen, 2005, p. 627). According to this suggestion, SMH offers a promising example of how human decision making might be explained in computational and neurophysiological terms. However, several recent criticisms charge SMH with vagueness and ambiguity (Colombetti, 2008; Dunn, Dalgleish, \& Lawrence, 2006). Indeed, one recent review identifies no fewer than 38 alternative interpretations of this hypothesis (Linquist \& Bartol, 2013). This discrepancy is perplexing. On the one hand, SMH is among the most influential theses to have emerged from cognitive neuroscience in recent decades, having informed innumerable discussions about the role of emotion in practical reasoning. On the other hand, it is unclear which functional roles somatic markers play in decision making (Ohira, 2010) and the hypothesis is difficult to state in precise terms that allow for experimental investigation (Dunn et al., 2006).

One possible defense of SMH is that, in practice, there is less ambiguity than might appear in theory. Previous critics identify several possible interpretations of SMH suggested over the course of its 25-year development. But perhaps, in practice, researchers converge on a 
particular interpretation. Previous criticisms overlook this possibility, because they focus on $\mathrm{SMH}$ as it is formulated by its chief proponents and not on the ways that it is interpreted by the scientific community. We begin by testing this hypothesis with a random sample of recent literature citing $\mathrm{SMH}$. Our findings suggest that interpretations of SMH are indeed highly variable. These findings underscore the need to understand and rectify the ambiguity problem.

We recast the ambiguity problem as a deficiency at the computational level - i.e. the intervening level between psychological descriptions and neurophysiological data. Focus on $\mathrm{SMH}$ has been on linking practical decision making deficits (e.g. poor financial decisions) to neurophysiological damage in the ventromedial frontal cortices, amygdalae, and other regions. A computational description ought to facilitate connection among these levels by specifying mechanisms underlying the deficit. The current computational account associated with SMH identifies a simple association/reactivation mechanism: somatic markers are linked to certain representations and then recalled during decision making. Psychological-level deficits are characterized as breakdown in this mechanism. The problem, we argue, is that the computational account does not explicate the psychological description because it fails to distinguish between different stages at which decision making might break down. This explains why SMH is so broadly construed. Adding a more detailed, multi-stage computational account of decision making allows one to generate and test more refined versions of SMH. Finally, our analysis provides a general lesson on the role of computational descriptions in bridging psychological and neurological accounts of cognition.

\section{Core Themes in Somatic Marker Research}

Given the multiplicity of competing interpretations of the somatic marker hypothesis a concise definition is perhaps impossible. However there are a core set of themes surrounding somatic marker research. These are briefly outlined in this section as a general orientation to the literature.

Most SMH researchers view emotions in the James-Lange tradition as representations of embodied changes in the autonomic nervous system. Somatic markers are brain states that "tag" or index such changes. At the same time these tags become associated with the representations, of objects or events in the world, that trigger them. Through such associations certain representations acquire emotional salience. After a childhood tussle with a porcupine, say, future thoughts of quilled quadrupeds might trigger an emotional response. An important corollary of this view is that somatic markers are sometimes reactivated without attendant physiological changes (Damasio, 1994). In this respect somatic markers serve both as triggers and as proxies for full blown emotional responses.

Another common theme in somatic marker research is that markers themselves are either positively or negatively valenced. Hence, the reactivation of a marker provides information about whether the associated object or event was experienced as "good" or "bad" on previous occasions. This information is thought (somehow) to enhance decision making.

Particular brain regions are implicated in the establishment and recollection of somatic markers. Ventromedial frontal cortices (VMF), amygdalae, and insular cortices are considered crucial in the formation and reactivation of somatic markers. Patients with VMF 
damage in particular have influenced the development of $\mathrm{SMH}$, as they exhibit both flattened affect and abnormal decision making abilities. Neurological details of SMH are outlined in more detail in Section 4.3.

The standard experimental probe behind $\mathrm{SMH}$ is the Iowa Gambling Task (IGT) (Bechara, Damasio, Damasio, \& Anderson, 1994). IGT is a card selection task in which participants must learn, over a series of selections, which of four decks is the most rewarding. Each selection earns a monetary reward or punishment. Decks are rigged so that those which are initially advantageous become deleterious, and vice versa, mid way through the game. Bechara and colleagues (1996) report that an autonomic (skin conductance) response predicts the switch to more rewarding decks. This finding is interpreted as evidence for a link between bodily feedback and decision making. This general hypothesis has been the subject of additional electrophysiological studies (Oya, Adolphs, Kawasaki, Bechara, \& Damasio, 2005) as well as studies of patients with VMF and amygdala damage (S. W. Anderson, Bechara, Tranel, \& Damasio, 1999; Bechara, Damasio, Damasio, \& Lee, 1999). The precise nature of this link, however, is a matter of ongoing controversy (Dunn et al., 2006; Gerrans, 2007; Maia \& McClelland, 2004).

As the discussion so far might reveal, SMH leaves open considerable room for interpretation. Most ambiguities arise in the attempt to explain in detail how somatic marker reactivation enhances decision making. We now turn to a survey of some competing views on this issue.

\section{How do Researchers Interpret SMH?}

How many alternate versions of the somatic marker hypothesis are there? Philosopher Giovanna Colombetti (2008) identifies two versions. "SMH-general" simply posits a role for emotions in decision making, while "SMH-specific" proposes that lesions to the VMF impair one's ability to form long term plans, or, "myopia for the future" (Bechara, Tranel, Damasio, \& Damasio, 1996). More recently, Linquist and Bartol (2013) have extended this analysis, identifying three independent axes along which versions of SMH equivocate. First, alternate versions differ in modality. That is, they disagree about whether somatic markers are necessary for decision making or merely contribute to this process. Second, alternate versions assign distinct processing roles to somatic markers. Sometimes markers are said to enhance the speed of decision making, while at other times they are thought to increase accuracy. Often they are said to do both. Finally, Linquist and Bartol identify multiple stages at which somatic markers potentially influence decision making. These can be broadly characterized into 'peripheral' and 'core' [Table 1]. The two peripheral stages occur at the initiation and termination of a decision process. Decision-point recognition is the initiation phase where an individual recognizes that a practical decision is called for. Execution is the termination stage where the subject follows through on a decision. Core stages can be divided into at least three: option generation involves the identification of alternative courses of action; deliberation involves inferring potential consequences from specific options; evaluation involves ranking or assigning value to each option. Linquist and Bartol (2013) demonstrate that the chief proponents of SMH have posited, at one time or another, a role for somatic markers in all five stages. However, equivocation among these alternatives is not explicit. 
Given the variety of possible interpretations of $\mathrm{SMH}$, an important empirical question concerns the way it is understood by working scientists. We selected five target presentations of SMH as the basis for our literature search.i These canonical presentations were published between 1991 and 2004 - the main period over which SMH was developed. They also represent a variety of publication venues: a bestselling book, articles in Science, Phil. Trans. Royal B, and Brain \& Cognition, and a chapter in an edited volume. An article was included in our sample if it referenced at least one of the five target presentations before September 2011. To further refine our search, articles were drawn from specific journals. Using the 'cited reference search' tool from Web of Science, we compiled lists of records citing the target articles, broken down by journal. We sought journals that appeared nearest the top across citation reports for all five target articles. Neuropsychologia appeared at or near the top most frequently; J Neuroscience also appeared with high fidelity. Both journals were included in our sample. Neuroimage and Brain \& Cognition also appeared at or near the top frequently, but were excluded. The former was excluded because of its highly specialist nature. The latter was excluded because early articles are not PDF-searchable, a pre-requisite for our coding methodology. Outside of neuroscience and the behavioural sciences, much of the discussion of SMH has come from philosophers publishing in philosophical and multidisciplinary humanities journals. This category accounted for the next largest source of citations to our target articles. Hence we included in our sample articles from Philosophical Psychology and J. Consciousness Studies, the two most prevalent sources of this type. Excluding duplicates and cases where SMH proponents (A. Damasio, H. Damasio, or A. Bechara) cited their own work left a representative pool of 103 articles by 337 authors.

A coding tool allowed us to identify alternative interpretations of SMH. For each instance in which one of the five canonical SMH articles was cited, the following information was recorded.

1. Does the author explicitly identify (a) a role for somatic markers in decision making, (b) a role for certain brain regions in decision making, (c) a role for somatic markers/ brain regions in determining performance on the Iowa Gambling Task (IGT), or (d) none of the above?

2. Does the author interpret $\mathrm{SMH}$ as a specific thesis about myopia for the future?

3. Does the author take somatic markers/ intact brain regions to be (a) necessary for, (b) involved in, or (c) play an unspecified role in decision making?

4. Does the author take somatic markers/intact brain regions to contribute to (a) speed, (b) accuracy, (c) both, or (d) an unspecified role in decision making?

5. Does the author specify the stage of decision making at which Somatic markers become engaged?

If there was an affirmative answer to the final question, we attempted to determine the stage at which somatic markers were being invoked, either as central/peripheral or according to the specific stage as outlined by Linquist and Bartol (2013) [Table 1]. Two people applied the coding scheme. To ensure uniformity, a series of practice rounds were undertaken on separate samples of articles similar to the ones that were coded. Coding of the SMH sample occurred after the third practice round, when concurrence among coders reached $>95 \%$. 
The first question functioned as a filter. Of the initial 103 articles, 59 were classified further, having invoked some version of SMH. More than half of these $(n=34)$ explicitly identified a role for somatic markers in decision making. Another 19 citations proposed a role for certain brain regions in decision making, while only 6 articles identified a role for brain regions/somatic markers in IGT performance. The 44 excluded articles invoked some other aspect of the author's work (e.g. experimental protocols).

Regarding the second question, nearly $24 \%$ of the citations to SMH interpreted it in accordance with Colombetti's (2008) SMH-specific. These authors took the SMH to imply that damage to brain regions involved in generating somatic markers results in myopia for the future.

Modal interpretations of the SMH also varied. Though $30 \%$ were unspecified, $56 \%$ held that somatic markers contributed to (some component of) decisions, while $12 \%$ interpreted them as necessary for decision making.

With regard to the fourth question about the contributions of somatic markers/ brain regions to decision making, $42 \%$ interpret the $\mathrm{SMH}$ as supporting a role for markers in accuracy, $12 \%$ both speed and accuracy, and $46 \%$ are unspecified. None of the articles in our sample interpreted the SMH strictly as a thesis about speed.

Few authors identified a specific stage at which somatic markers influence decision making, with $51 \%$ of the answers to question 5 coming out negative. Of those that did specify a role $(n=29), 7 \%$ of the articles favoured a deliberative role for somatic markers in the identification of consequences. Another $12 \%$ saw somatic markers playing an evaluative role in ranking or assessing options. Only $3 \%$ assigned a role for somatic markers in execution. Interestingly, $25 \%$ of the articles evidenced an ambiguous reference to core stages, vacillating between one or more sub-processes (see Figure 1). 
To summarize, there appears to be no consensus in our sample about the meaning of the somatic marker hypothesis. Even on such fundamental questions as modality and processing role, there is a diversity of alternative interpretations. It is noteworthy that not a single article appealed to speed as the sole processing role for somatic markers; all authors who took somatic markers as contributing to speed assumed that they simultaneously enhance accuracy. This is surprising, given that these properties are likely to trade off against one another. On the more nuanced question about the specific stage of decision making at which markers have a role, more than half of the authors in our sample expressed no commitment. This suggests that most authors are not thinking about SMH in the context of a detailed computational account of decision-making. This reading is further supported by Question 1 in our survey, in which $39 \%$ of the authors described SMH purely in the context of brain regions, not mentioning somatic markers at all. A sizable proportion of articles equivocated among alternative stages. Among those who did commit to a specific stage of decision making for somatic markers, opinions were split mainly between deliberation and evaluation. Only two authors interpret the SMH as suggesting a role in execution. This is also surprising, given that several authors have singled out execution as a particularly promising role for somatic markers (Dunn et al., 2006; Linquist \& Bartol, 2013).

As mentioned, our sample aimed to capture the primary disciplinary perspectives engaged in SMH research. It is possible that a greater number of articles, perhaps from a narrower range of journals, including journals from a more specific subset of disciplines, might reveal greater consensus. However, none of our observations suggest that this should be so. Our findings do not suggest that researchers are entirely unaware of ambiguity. Four of the articles in our survey demonstrated some awareness of the fact that results on the IGT are open to multiple interpretations; though zero articles demonstrated an awareness of ambiguity in SMH itself.

This sample suggests that the ambiguity found in formulations of $\mathrm{SMH}$ has resulted in a spectrum of interpretations of the theory's central claims. As we shall now argue, such widespread ambiguity can be explained by the lack of an adequate computational-level model of practical decision making.

\section{Three Levels of Description in SMH}

Levels of analysis in cognitive theory have been widely discussed (J. R. Anderson, 1990; Dennett, 1987; Marr, 1982; Newell, 1990; Pylyshyn, 1984; Stanovich, 1999; Sun et al., 2005). The array of different schemes for describing these levels can be 'bewildering', as one researcher has warned (Sterelny, 1990); and it is not our aim to review this literature here. What follows is a brief outline of the three-fold distinction between psychological, computational, and implementation levels. In each case we identify the corresponding descriptions in SMH at each level.

\section{i. Psychological level Descriptions of SMH}

Here we understand a psychological description to consist of three features. First, it describes some functional capacity exhibited by a whole system and not just some of its parts (Pylyshyn, 1984). Second, a psychological description identifies intentional states in the system 
that it describes (Dennett, 1987; Stanovich, 1999). Finally, it assumes that the agent is rational (J. R. Anderson, 1990; Pylyshyn, 1984) in the sense that subjects are expected to use available information in ways that accord with their goals.

Psychological descriptions of the somatic marker hypothesis identify a vague role for emotions in practical reasoning. These descriptions are typically framed against contrast cases in which decision making falters in brain damaged patients. Most notorious among these is Damasio's focal patient, Elliot, who exhibited severe practical deficits after undergoing bilateral ablation to VMF (see Damasio, Tranel, \& Damasio, 1991; Damasio, 1994; Eslinger \& Damasio, 1985). Some of Elliot's behavioural anecdotes suggest, at the psychological level, ways that emotion might influence decision making in less impaired subjects.

Perhaps Elliott's most widely cited deficit is chronic indecisiveness. For example:

He needed about two hours to get ready for work in the morning, and some days were consumed entirely by shaving and hair-washing. Deciding where to dine might take hours, as he discussed each restaurant's seating plan, particulars of each menu, atmosphere and management. He would drive to each restaurant to see how busy it was, but even then he could not finally decide which to choose. Purchasing small items required in-depth consideration of brands, prices, and the best method of purchase. (Eslinger \& Damasio, 1985, p. 1732)

Such anecdotes call attention to the relative economy of normal decision making. Elliot seems unable to limit the time and effort invested in decisions. He is unable to manage the number of options and the breadth of information considered. These deficiencies can perhaps be portrayed functionally as an inability to limit attention to the relevant facets of a prevailing situation (Damasio, 1994). But it is also possible that Elliott is simply unable to break out of the decision-making loop (Linquist and Bartol, 2013). Indeed, other anecdotes suggest that Elliot had no problem making decisions per se. Where he faltered was in bringing himself to follow through with an execution. Elliot self-reports that, even after successfully generating and evaluation lists of options: "I still would not know what to do" (Saver \& Damasio, 1991, p. 1246). It is conceivable that this lack of "oomph," rather than some defect in the deliberation process itself, inspires Elliot to continually search for different features of a situation that might (eventually) propel him into action (Eslinger \& Damasio, 1985).

A second type of deficit is also noteworthy. Consider the following:

[Elliot's] social conduct was profoundly affected by his brain injury. Over a brief period of time, he entered disastrous business ventures (one of which led to predictable bankruptcy), and was divorced twice (the second marriage, which was to a prostitute, only lasted 6 months). He has been unable to hold any paying job since the time of surgery, and his plans for future activity are defective. (Damasio, Tranel, \& Damasio, 1990, p. 82)

In these accounts Elliot appears able to execute decisions, but does so in foreseeably disastrous ways. Such descriptions of Elliot's poor choices support the 'myopia for the future' interpretation, which suggest that normal decisions accord with an agent's long-term goals.

To summarize, psychological descriptions of Elliot's cognitive deficits draw attention to a cluster of features characteristic of normal decision making. These include:

Economy in the time and effort required to reach a decision.

Relevance of considerations to appropriate contexts.

Execution of decisions once they have been made.

Foresight to take into account likely undesirable outcomes. 
Psychological-level descriptions of SMH assume that somatic markers are beneficially related to (at least some of) these core features.

\section{ii. Computational Models of SMH}

Most multi-level accounts of cognition identify a computational (also called algorithmic) level below the psychological level (cf. Marr, 1982; Sun \& Franklin, 2006). A computational model describes a series of steps that execute some system-level capacity. Often, these models identify distinct structures (e.g. symbols or subprocesses) not mentioned at the psychological level. A particular system will admit of multiple computational descriptions that vary in the number and specificity of the steps or structures that they identify. The level of detail might range from simple box-and-arrow process maps to more complex computer-implemented cognitive architectures.

The computational model behind $\mathrm{SMH}$ is, at base, a simple account of the association between somatic markers and representations and their subsequent re-activation. The model holds that a negative or positively valenced state - a somatic marker - becomes attached to a mental representation of a stimulus. This happens through experience. These valenced markers are subsequently re-activated along-side those representations. In virtue of their negative/positive valence somatic markers convey information about the stimulus, thereby influencing the agent's behaviour. More specific versions of this base mechanism link working memory to valence (Damasio, 1994), proposing that strongly marked representations will be held in working memory as a way of focussing attention on salient stimuli.

\section{iii. Implementation Level Descriptions of SMH}

Implementation level models attempt to capture the neurophysiological components and processes underlying cognition. These vary in anatomical specificity, ranging from broadly characterized neurological regions to specific descriptions of cellular and sub-cellular processes. There is no simple or single answer to the question about the right amount of anatomical detail to include in an implementation-level model. It will often depend on a researcher's goals.

The neurophysiological description of SMH has been praised as among its strengths (Dunn et al., 2006). The sequences of anatomical events behind the formation and reactivation of somatic markers has been specified in considerable detail, relying on both human and animal studies. The amygdala is thought to be a crucial 'trigger' structure in the initial formation of somatic markers (Bechara, 2003). Damasio and Bechara (2005) explain that the amygdala marries a stimulus to a somatic state. The stimulus is processed via the thalamus, while the somatic state is evoked via the hypothalamus and autonomic brainstem nuclei, which in turn produce changes in other visceral structures and the central nervous system (Bechara, 2004). Once linked, a pattern of activation is formed. After initial formation, the authors claim that this same pattern is reactivated upon subsequent encounters with the stimulus or its representation (eg. in memory or imagination). In cases of re-activation, the VMF, rather than the amygdala, acts as the trigger structure. It couples mental representations of a stimulus to the previously-established somatic state pattern (Bechara, Damasio, \& Damasio, 2000). Because some somatic state activation seems to occur without detectable 
changes to the central nervous system, SMH specifies a second process in which the somatic state re-activation bypasses the central nervous system, acting instead on brain regions normally involved in motor pathways and somatosensory responses. This system - dubbed the 'as if body loop (Bechara, 2004) - produces a neural state change that mimics an absent bodily response. This is connected with work on self-awareness and the insula. A growing body of research suggests that the insula is involved in representing body states, which may be integrated into various cognitive and pre-cognitive processes, like choice and decision making (Craig, 2009).

Additionally, Bechara and colleagues (2001) have probed the contributions of certain neurotransmitter systems to decision making behaviours. Following this work, SMH includes an account explaining how linking neurotransmitter control to somatic state re-activation could provide a mechanism for modulating synaptic activity, raising/lowering the action potentials of neuronal groups underlying behaviour and cognition (see Bechara \& Damasio, 2005).

\section{Ambiguities in SMH Owe to a Disconnect Between Psychological and Computational Levels}

Having separated distinct levels of description in $\mathrm{SMH}$, perhaps the most striking feature is the way in which the simple associative computational mechanism has been investigated and characterized at the implementation level. This exemplifies fruitful investigation across these two levels. Neuroanatomical research has revealed distinctions invisible at the computational level. For example, the fact that the VMF replaces the amygdala as the trigger structure after initial pattern formation reveals that initial activation and subsequent re-activation are in fact different types of process. This shows how implementation-level details help flesh out and expand computational-level mechanisms.

The same relationship does not currently obtain between psychological and computational or neurological levels. At the psychological level one finds a general characterization of some core features of decision making that are absent in VMF patients. This tells us that somatic markers aid decision making, but not how they do so. This explanation cannot come from the implementation level, either, since it is unclear what processes to search for in the brain. Categories like 'decision making' and 'economy' and 'relevance' are far too broad to explicate in neurological terms.

Enter the computational level, which ought to serve as a bridge between psychological processes and neurological details. Computational descriptions can explicate mechanisms that may lay behind broad psychological processes. Those mechanisms can then be investigated at the implementation level. In order to bring psychological claims into the fold, the computational mechanism must be sufficiently detailed to unpack and expand the psychological description. However the basic association/re-activation model currently associated with SMH is unfit for this purpose. Though it specifies how and when somatic markers may be formed and activated, it does not address how this association and reactivation participates in practical decision making. There is a disconnect between the psychological claims and the computational mechanism. This disconnect is the source of the ambiguity problem. 
To illustrate, take the property of economy. Tests on Elliot and other brain lesion patients suggest a relationship between economy and somatic markers. Yet there are a number of plausible ways in which the association/reactivation mechanism could function to aid decisions in this way. Normal decision making might be economical because somatic markers streamline the range of options up for consideration, restricting focus only to those which are positively valenced. Alternatively, somatic markers might assign some kind of weighting function to the evaluation of candidate options, perhaps ranking them according to net valence. This interpretation is arguably at play in IGT (Colombetti, 2008; Dunn et al., 2006; Maia \& McClelland, 2004; Tomb, Hauser, Deldin, \& Caramazza, 2002). A third possibility is that somatic markers enhance economy by highlighting certain information about certain options, for example, drawing attention to consequences. A fourth possibility is that somatic markers index the relevance or gravity of a potential outcome for the subject, and influence the amount of time or care dedicated to cost benefit analysis. Somatic markers are conceivably involved in any one of these processes.

Similar possibilities arise when attempting to explain the relevance, foresight, and execution of normal decision making. These are very general properties of a multi-stage process. The basic association/reactivation mechanism leaves too many options for explaining how that mechanism might interact with decision making to produce these psychological properties.

What is needed is a way to regiment and align computational and psychological accounts. To this end, we offer the following five-step model of decision making. For simplicity, we present these as 'stages', but they are best conceived as alternate mechanisms or subroutines within the decision making process - it seems likely that these stages would occur in parallel and with feedback loops. We offer this simple idealization as a first pass for developing a computational model that will help to generate a range of specific hypotheses about how a somatic marker mechanism might be employed in the service of practical decision making.

This five-stage decision making model allows for regimentation of the various possible computational hypotheses, in the manner demonstrated above. In laying out the possible functional roles for somatic markers this model should also point to the kinds of bodily state mapping that are necessary to fill certain roles. This is necessary in order to explain how somatic markers can fill the various functional roles ascribed to them. Recent research has identified other brain structures besides the VMF and amygdala as potentially instrumental to decision making. For instance, philosopher Phil Gerrans (2007) argues that the dopamine system both confers salience on certain stimuli and also motivates agents to pursue rewarding goals. Gerrans argues that this system of dopamine-based reward, rather than the assessment of valence, drives decision making on tasks like the IGT. This is an important challenge to $\mathrm{SMH}$. However, it inherits many of the same ambiguities. What is the precise role that dopamine plays in the decision making process? The suggestion is that dopamine influences both salience and motivation. However there are several ways in which salience might aid decisions. Perhaps salience aids in the quick generation or elimination of options. Perhaps it renders options more/less likely to be chosen upon evaluation. A more detailed model of decision making helps to tease apart these challenges to $\mathrm{SMH}$ as much as it helps to identify 


\begin{tabular}{|c|c|c|c|}
\hline & Stage & Psychological description & $\begin{array}{l}\text { Possible Computational } \\
\text { description }\end{array}$ \\
\hline & $\begin{array}{l}\text { I. Decision- } \\
\text { point } \\
\text { recognition }\end{array}$ & $\begin{array}{l}\text { Subject diverts attention away } \\
\text { from other activities and on the } \\
\text { task of decision making. }\end{array}$ & $\begin{array}{l}\text { Representations provide a cue that } \\
\text { a practical decision is called for, } \\
\text { and the associated somatic marker } \\
\text { acts as an "alarm bell" in } \\
\text { redirecting attention. }\end{array}$ \\
\hline \multirow{4}{*}{ 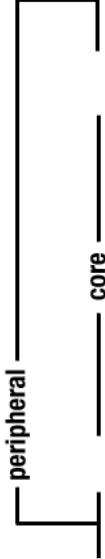 } & $\begin{array}{l}\text { 2. Option } \\
\text { generation }\end{array}$ & $\begin{array}{l}\text { Subject identifies a range of } \\
\text { alternative courses of action. }\end{array}$ & $\begin{array}{c}\text { Somatic marker acts as an "anchor" } \\
\text { linking together representations of } \\
\text { related options, allowing for rapid } \\
\text { co-activation. }\end{array}$ \\
\hline & 3. Deliberation & $\begin{array}{l}\text { Subject infers a limited set of } \\
\text { consequences for each } \\
\text { candidate option. }\end{array}$ & $\begin{array}{l}\text { Somatic markers facilitate recall of } \\
\text { most emotionally-salient options. }\end{array}$ \\
\hline & 4. Evaluation & $\begin{array}{l}\text { Subject assigns value to } \\
\text { candidate options. }\end{array}$ & $\begin{array}{c}\text { Somatic markers transmit valence } \\
\text { to representations of options by } \\
\text { association. }\end{array}$ \\
\hline & 5. Execution & $\begin{array}{l}\text { Subject acts in accordance } \\
\text { with the most valued option. }\end{array}$ & $\begin{array}{l}\text { Somatic markers motivate by } \\
\text { rewarding action or punishing } \\
\text { inaction }\end{array}$ \\
\hline
\end{tabular}

Table I. Multi-stage model of practical decision making. For each stage, a psychological-level description is accompanied by an example computationallevel hypothesis, proposing a role for somatic markers at that stage.

candidate versions of the hypothesis. A similar point applies to the recent finding that the anterior cingulated cortex is involved in the assignment of hedonic tone to a stimulus (Craig, 2009; Phillips, Drevets, Rauch, \& Lane, 2003; Sescousse, Redouté, \& Dreher, 2010). It might be argued that this brain structure, which presumably does not transmit valence, but assigns other qualitative features to a stimulus, is involved in the general process of decision making. Our five step model provides a framework for refining and testing this hypothesis.

In the final section of this paper we outline some of the methodological implications of this model on experimental design. Before doing so, it is important to draw a connection between our computational model of decision making and existing computational models of $\mathrm{SMH}$.

Often the aim of computational modelling is to create a complex architecture, implemented on an electronic computer, that performs certain (often circumscribed) tasks in ways similar to humans (Sun et al., 2005). Existing computer models of SMH are problematic because they assign high-level psychological functions (e.g. myopia for the future) to specific brain regions. They are also problematic because they rely on the IGT as their sole decision making probe (Kalidindi, Bowman, \& Wyble, 1994; Wagar \& Thagard, 2004). The most notable of these models is Wagar and Thagard's (2004) GAGE neural network (named after Phineas Gage the railroad engineer). GAGE uses collections of artificial neurons to represent distinct brain regions, such as the VMF, amygdala, and hippocampus. In order to assign roles to various brain structures, models like GAGE must rely on existing psychological and computational descriptions. In determining the profile for the VMF, for instance, GAGE's creators relied on the contentious 'myopia for the future' interpretation of SMH. The VMF was accordingly assigned the role of encoding preference for future outcomes. Since this hypothesis is unclear and as-yet untested (Dunn et al., 2006), GAGE does not tell us what role 
somatic markers play. It tells us only that an impaired ability to anticipate future outcomes results in poor IGT performance. Setting aside the use of the IGT as a probe for SMH, these models still do not yet provide insight into somatic marking. We cannot assess whether GAGE reflects somatic marking in human cognition because we do not yet know how somatic markers influence particular stages of decision making. GAGE, and other cognitive architectures for decision making, provide hope for future modelling of SMH. But this level of modelling is possible only once specific computational models are hypothesized, tested, and confirmed.

\section{Implications for Experimental Design}

The general claim that emotions or somatic markers are associated with decision making appears to be well-established (Dunn et al., 2006). Future work ought to investigate how they are implicated. Earlier it was noted that IGT is currently the primary experimental probe for SMH. Our five stage model helps to reveal why it is unfit for this purpose. The IGT cannot test whether somatic markers are involved in decision-point recognition because decision points are artificially delineated by the experimental setup. It similarly cannot test the thesis that somatic markers assist in option generation because the options are pre-selected. The IGT cannot test the claim that somatic markers highlight relevant information about the options, as participants are required to consider only one type of information: expected profit. The IGT is also ill-equipped to test the thesis that somatic markers are involved in motivating execution of a decision because selections are explicitly motivated as part of the experimental setup. In sum: the IGT is not a test of specific computational models. The IGT is a test of the broad psychological claim that affect is implicated in practical decision making. At most the IGT can be used to determine whether specific neurological structures contribute (somehow) to decision making. But the IGT is a rather blunt instrument insofar as it skips the computational level altogether.

Linquist \& Bartol (2013) have argued that some early experiments developed by Saver and Damasio (1991), later abandoned in favour of the IGT, were in fact superior robes of specific stages in decision making. Here we elaborate these suggestions and propose some additional methods for investigating peripheral stages in practical decision making.

The Optional Thinking Test measures participants' ability to generate candidate solutions to hypothetical social dilemmas. This is a straightforward test of option generation. Saver and Damasio (1991) compared the performance of a VMF lesion patient on the Optional Thinking Test to controls. Although they found no significant difference, this study was performed on only a single subject (Elliott). To our knowledge this procedure has not been tested on a larger sample. A second strategy would deploy physiological measurements of autonomic arousal to determine whether they correlate with the rate or number of options generated in this task. Functional imaging might offer a third strategy for identifying the brain structures involved in option generation. Attention to the nature of this process might recommend focus on regions associated with salience, such as the PACC, or valence, such as the amygdala (Phillips et al., 2003).

In their Awareness of Consequences Test, Saver and Damasio (1991) presented participants with four hypothetical predicaments that offer a temptation to transgress certain 
social norms. Participants were asked to explain which considerations they would take into account when deciding what to do. This task is an excellent probe for deliberation, that is, for the ability to generate foreseeable consequences from available options. Interestingly, Elliot performed significantly better than controls on this task, suggesting that an intact VMF is not necessary for this task. Again, a larger sample would provide more conclusive findings. Physiological recordings, correlated with performance measures in the rate or number of consequences identified, would further probe whether somatic markers facilitate either the speed or the accuracy of this stage in decision making. Anatomical focus might be directed at regions associated with salience, such as the PACC (Phillips et al., 2003).

A third task developed by Saver and Damasio (1991) is the Means/Ends Problem-Solving Test. This story completion task asks participants to generate a step-by-step plan for achieving some social objective; for example, the goal of making friends in a new neighbourhood. Scoring is based on the number of relevant versus irrelevant factors that participants generate. This task is particularly well suited for testing any computational model that predicts somatic markers participate in deliberation.

The hypothesis that somatic markers influence evaluation predicts that markers will transmit valence onto alternative courses of action. A simple test might draw on Jonathan Haidt's paradigm for testing the influence of emotion on moral reasoning (Schnall, Haidt, Clore, \& Jordan, 2008). In this task, participants are presented with disgust-evoking stimuli while rating the severity of moral transgressions. He finds that a strong disgust response accentuates moral condemnation. In a decision making context participants might be presented with candidate solutions to some practical challenge, and asked to rate them for their practicality. If negative somatic markers transmit their valence onto associated representations, then practicality rankings should be lower in the disgust-inducing context than in controls. Similar experiments using a positive stimulus as the independent variable could likewise test whether positive valence is transmitted in the evaluation of candidate courses of action. Such experiments could further incorporate a physiological or neurological dimension. Given that the nature of evaluation recommends a role for hedonic tone, focus should be directed toward the ACC (Craig, 2009; Sescousse et al., 2010)

Suggestions up to this point have focussed on experiments probing the core stages of decision making. It is more challenging to devise experiments that probe peripheral stages decision-point recognition and execution. Our suggestions are therefore tentative.

Decision point recognition is inherently a refocusing of one's attention, often away from some engaging activity, onto the task of decision making. An adequate experimental probe might first engage participants' attention, and then present emotionally evocative stimuli as the independent variable. Video games are well suited to this purpose. We are presumably all familiar with games that require players to find their way through a maze. Emotionally salient distractions could be built into the fabric of the game. The hypothesis that somatic markers facilitate decision-point recognition predicts that emotional stimuli will (a) disengage a subject from an otherwise engrossing activity and (b) redirect attention towards the identification of alternative options. As a dependent variable, the game could include a function that enables players to request practically relevant information on demand. One would expect such requests to be triggered by evocative stimuli. 
The key is to isolate this final stage of the decision process from ones that precede it. Thus, participants would have to indicate when a decision to execute some action has been reached. One could then measure latency in execution following a decision. Of particular interest is the possibility that failure to execute a decision reinitiates the process, perhaps giving rise to the sort of chronic indecisiveness that sometimes plagues Elliot.

\section{Conclusion}

A promising explanation for the proliferation of somatic marker hypotheses is that, though researchers are attuned to the broad psychological claim that emotions play a role in practical decision making and to the interesting way in which the association/re-activation mechanism has been explained at the implementation level, they are generally not attuned to the relationship between the computation and the psychological levels. We hope our analysis has suggested a route forward. For research on decision making, our work suggests that a simple computational model will help highlight and regiment ambiguity within a more general psychological account. Some readers might be surprised at just how simple our computational model of decision making is, in fact. Presumably an even more detailed model could generate even more precise hypotheses.

\section{References}

Anderson, J. R. (1990). The adaptive character of thought. Hillside, N.J.: Erlbaum.

Anderson, S. W., Bechara, A., Tranel, D., \& Damasio, A. R. (1999). Impairment of social and moral behaviour related to early damage in human prefrontal cortex. Nature Neuroscience, 2(II), 1032-1037.

Bechara, A. (2003). Role of the Amygdala in Decision-Making. Annals of the New York Academy of Sciences, 985, 356-369.

Bechara, A. (2004). The role of emotion in decision-making: evidence from neurological patients with orbitofrontal damage. Brain and Cognition, 55(I), 30-40. doi:10.10I6/j.bandc.2003.04.00 I

Bechara, A., \& Damasio, A. R. (2005). The somatic marker hypothesis: A neural theory of economic decision. Games and Economic Behavior, 52(2), 336-372. doi:10.1016/j.geb.2004.06.010

Bechara, A., Damasio, A. R., Damasio, H., \& Anderson, S. W. (1994). Insensitivity to future consequences following damage to human prefrontal cortex. Cognition, 50(I-3), 7-I5.

Bechara, A., Damasio, H., \& Damasio, A. R. (2000). Emotion, decision making and the orbitofrontal cortex. Cerebral Cortex, 10(3), 295-307.

Bechara, A., Damasio, H., \& Damasio, A. R. (200I). Manipulation of dopamine and serotonin causes different effects on covert and overt decision-making. Society for Neuroscience Abstracts, 27, 126.

Bechara, A., Damasio, H., Damasio, A. R., \& Lee, G. P. (1999). Different contributions of the human amygdala and ventromedial prefrontal cortex to decision-making. The Journal of Neuroscience, 19(13), 5473-8I. 
Bechara, A., Tranel, D., Damasio, H., \& Damasio, A. R. (1996). Failure to respond autonomically to anticipated future outcomes following damage to prefrontal cortex. Cerebral Cortex, 6(2), 2I5-25.

Colombetti, G. (2008). The Somatic Marker Hypotheses, and What the lowa Gambling Task Does and Does not Show. The British Journal for the Philosophy of Science, 59(I), 5I-7I. doi:10.1093/bjps/axm045

Craig, A. D. B. (2009). How do you feel - now? The anterior insula and human awareness. Nature Reviews Neuroscience, I0(January), 59-70.

Damasio, A. R. (1994). Descartes' Error: Emotion Reason and the Human Brain. NY: Putnum.

Damasio, A. R., Tranel, D., \& Damasio, H. (1990). Individuals with sociopathic behavior caused by frontal damage fail to respond autonomically to social stimuli. Behavioural Brain Research, 4I(2), 8I-94.

Damasio, A. R., Tranel, D., \& Damasio, H. (I99I). Somatic markers and the guidance of behavior: Theory and preliminary testing. In S. Levin, M. Eisenberg, \& A. L. Bendon (Eds.), Frontal Lobe Function and Dysfunction. Oxford University Press.

Dennett, D. (1987). The Intentional Stance. Cambridge: MIT Press.

Dunn, B. D., Dalgleish, T., \& Lawrence, A. D. (2006). The somatic marker hypothesis: a critical evaluation. Neuroscience and Biobehavioral Reviews, 30(2), 239-7I. doi:10.1016/j.neubiorev.2005.07.00I

Eslinger, P. J., \& Damasio, A. R. (1985). Severe disturbance of higher cognition after bilateral frontal lobe ablation: patient EVR. Neurology, 35(I2), I73I-4I.

Gerrans, P. (2007). Mental time travel, somatic markers and "myopia for the future." Synthese, I59(3), 459-474. doi: I0.1007/s | |229-007-9238-x

Kalidindi, K., Bowman, H., \& Wyble, B. (1994). An Investigation of the Myopia for the Future Consequences Theory of VMF Patient Behaviour on the lowa Gambling Task; An Abstract Neural Network Simulation. Progress in Neural Processing, 33 I-338.

Linquist, S., \& Bartol, J. (2013). Two Myths about Somatic Markers. British Journal for the Philosophy of Science, 64(3), 455-484. doi:10.1093/bjps/axs020

Maia, T. V, \& McClelland, J. L. (2004). A reexamination of the evidence for the somatic marker hypothesis: what participants really know in the lowa gambling task. Proceedings of the National Academy of Sciences of the United States of America, I0I(45), I6075-80. doi:I0.1073/pnas.0406666I0 I

Marr, D. (1982). Vision. San Francisco: W. H. Freeman.

Newell, A. (1990). Unified Theories of Cognition. Cambridge: Harvard University Press.

Ohira, H. (2010). The Somatic Marker Revisited: Brain and Body in Emotional Decision Making. Emotion Review, 2(3), 245-249. doi:I0.I I77//7540739/0362599

Oya, H., Adolphs, R., Kawasaki, H., Bechara, A., \& Damasio, A. R. (2005).

Electrophysiological correlates of reward prediction error recorded in the human prefrontal cortex. Proceedings of the National Academy of Sciences of the United States of America, 102(23), 835I-8356. 
Phillips, M. L., Drevets, W. C., Rauch, S. L., \& Lane, R. (2003). Neurobiology of emotion perception I: the neural basis of normal emotion perception. Biological Psychiatry, 54(5), 504-5I4. doi:10.1016/S0006-3223(03)00168-9

Pylyshyn, Z. (1984). Computation and Cognition. Cambridge, MA: MIT Press.

Saver, J., \& Damasio, A. R. (199I). Preserved Access and Processing of Social Knowledge in a patient with acquired sociopathy due to ventromedial frontal damage. Neuropsychologia, 29(I2), |24I-1249.

Schnall, S., Haidt, J., Clore, G., \& Jordan, A. (2008). Disgust as Embodied Moral Judgment. Personality and Social Psychology Bulletin, 34, I096-I 09.

Sescousse, G., Redouté, J., \& Dreher, J.-C. (2010). The architecture of reward value coding in the human orbitofrontal cortex. The Journal of Neuroscience, 30(39), I3095-104. doi:I0.1523/JNEUROSCI.350I-10.2010

Stanovich, K. E. (1999). Who is rational? Studies of individual differences in reasoning. Mahwah, NJ: Erlbaum.

Sterelny, K. (1990). The representational theory of mind: An introduction. Oxford: Basil Blackwell.

Sun, R., Coward, L. A., \& Zenzen, M. J. (2005). On levels of cognitive modeling. Philosophical Psychology, I8(5), 6I3-637. doi:10.1080/095।5080500264248

Sun, R., \& Franklin, S. (2006). Computational Models of Consciousness: A Taxonomy and Some Examples. In P. D. Zelazo, M. Moscovitch, \& E. Thompson (Eds.), Cambridge Handbook of Consciousness (pp. I5I-174). Cambridge University Press.

Tomb, I., Hauser, M., Deldin, P., \& Caramazza, A. (2002). Letters to the editor. Nature Neuroscience, 242(I I), I473-7. doi:I0.2460/javma.242.I I.I473

Wagar, B. M., \& Thagard, P. (2004). Spiking Phineas Gage: a neurocomputational theory of cognitive-affective integration in decision making. Psychological review, III(I), 67-79. doi:10.1037/0033-295X.III.1.67 


\section{Endnotes}

i Bechara, A. [2004]: 'The role of emotion in decision-making: Evidence from neurological patients with orbitofrontal damage', Brain and Cognition, 55:I, 30-40.

Damasio, A. R. [ 1 994]: Descartes' Error: Emotion, Reason, and the Human Brain, New York: Putnam.

Damasio, A. R., Tranel, D. and Damasio, H. [1991]: 'Somatic markers and the guidance of behavior: Theory and preliminary testing'. In H.S. Levin, H.M. Eisenberg and Bendon, A.L. (eds.), Frontal Lobe Function and Dysfunction, Oxford, UK: Oxford University Press

Damasio, A. R. [ 1996]: 'The somatic marker hypothesis and the possible functions of the prefrontal cortex', Philosophical Transactions of the Royal Society of London, 35 I, I4 I 3-20

Bechara, A., Damasio, H., Tranel, D. and Damasio, A. R. [ 1997 ]: 'Deciding advantageously before knowing the advantageous strategy', Science, 275, 1293-95. 